\title{
FIRST IN-SITU OBSERVATIONS OF NEUTRAL AND PLASMA DENSITY FLUCTUATIONS WITHIN A PMSE LAYER
}

Franz-Josef Lübken and Gerald Lehmacher

Department of Physics, Bonn University, Bonn, Germany

Tom Blix, Ulf-Peter Hoppe and Eivind Thrane

Norwegian Defence Research Establishment, Kjeller, Norway

John Cho and Wesley Swartz

Cornell University, Ithaca, New York, USA

\begin{abstract}
The NLC-91 rocket and radar campaign provided the first opportunity for high resolution neutral and plasma turbulence measurements with simultaneous observations of PMSE (Polar Mesospheric Summer Echoes). During the flight of the TURBO payload on August 1, 1991, CUPRI and EISCAT observed double PMSE layers located at 86 and $88 \mathrm{~km}$ altitude, respectively. Strong neutral density fluctuations were observed in the upper layer but not in the lower layer. The fluctuation spectra of the ions and neutrals within the upper layer are consistent with standard turbulence theories. However, we show that there is no neutral turbulence present in the lower layer and that something else must have been operating here to create the plasma fluctuations and hence the radar echoes. Although the in situ measurements of the electron density fluctuations are much stronger in the lower layer, the higher absolute electron density of the upper layer more than compensated for the weaker fluctuations yielding comparable radar echo powers.
\end{abstract}

\section{Introduction}

Strong VHF radar echoes are frequently observed in the polar summer upper mesosphere, a phenomenon known as 'polar mesosphere summer echoes' (PMSE) [Ecklund and Balsley, 1981; Röttger et al., 1988; Hoppe et al., 1988]. It was realized earlier that it is difficult to explain the PMSE echoes by the traditional theory of backscatter from turbulent structures in the electron gas. The reason for this is that the spatial extent of the density inhomogeneity must be in the order of the radar half-wavelength ( $3 \mathrm{~m}$ and 0.67 $m$ for 50 and $224 \mathrm{MHz}$ radars, respectively). These very small scales are normally located in the viscous subrange of the spectrum, where molecular diffusion destroys fluctuations caused by turbulent cascading. Only unrealistically strong turbulence could support these small scales against molecular diffusion. Kelley et al. [1987] suggested that the electrons may not be good tracers of neutral air turbulence because they are coupled to the ions, which in turn can exhibit smaller structures than the neutrals, provided their

Copyright 1993 by the American Geophysical Union.

Paper number 93GL00851

0094-8534/93/93GL-00851\$03.00 diffusivity is significantly reduced due to unusually high masses caused by clustering. This process is supported by the very low temperatures in the summer mesopause region. In the theory of a passive scalar turbulent spectrum a low molecular diffusivity, $D_{\theta}$, of a passive tracer $\theta$ results in a high Schmidt number defined as $S c=\nu / D_{\theta}(\nu$ $=$ kinematic viscosity).

Mechanisms to create very small structures in the electron gas other than turbulence have been discussed in the literature. For example, Röttger and LaHoz [1990] have proposed the creation of steep electron density gradients due to the enhanced recombination coefficient of electrons with clustered positive ions. If these gradients are steep enough (significant changes over a spatial distance of $\lambda_{\text {radar }} / 2$ ) they result in Fresnel backscattering. Some weak background turbulence may cause mixing of these steep gradients, which in turn increases the scatter cross section. Experimental evidence for a non-turbulent backscatter mechanism was found from in situ and radar measurements [Ulwick et al., 1988; Röttger et al., 1988].

In order to better understand the role of turbulence in the creation of PMSE echoes, reliable in situ measurements of neutral air and plasma turbulence together with simultaneous observations of PMSE were needed. Such measurements were performed during the recent NLC-91 campaign. First results are presented in this paper and in the companion papers in this issue.

\section{Instrumental}

The 'Noctilucent Cloud' campaign (NLC-91) was conducted at Esrange $\left(67.9^{\circ} \mathrm{N}, 21.1^{\circ} \mathrm{E}\right)$, North Sweden, in August 1991. The scientific objectives and the measurements performed in situ and by radars are described in other papers in this issue. Here we will mainly discuss data from the TURBO payload launched on August 1, 1991 at 01:40:00 UT ('Salvo B'). The payload name 'TURBO' indicates that it is primarily designed to measure turbulence. The payload is equipped with sensors to measure neutral (TOTAL, downleg), positive ion (PIP, up- and downleg) and electron densities (EPIP, downleg). More details can be found elsewhere [Hillert et al., 1992; Blix et al., 1990]. Background temperatures were measured by falling spheres, one of which was launched $44 \mathrm{~min}$ after TURBO.

Concerning small scale fluctuations, the data reduction is similar for all three probes: relative density fluctuations 
$\left(n(z)-n_{\text {ref }}\right) / n_{\text {ref }}$, also called 'residuals' $r(t)$, are deduced from the measured density profile $n(z)$ and from a reference profile $n_{\text {ref }}$, obtained by smoothing the density profile. A spectral analysis of the time series $r(t)$ is performed and quantitative turbulent parameters, such as turbulent energy dissipation rates $\epsilon$, are derived [Lübken, 1992].

Two VHF radars were operational during the NLC-91 campaign: the Cornell University Portable Radar Interferometer ('CUPRI') at $46.9 \mathrm{MHz}$ and the EISCAT VHF radar at $224 \mathrm{MHz}$. Technical details can be found elsewhere [Providakes et al., 1983; Hoppe et al., 1988]. CUPRI was located close to the rocket launch site. Still, there is a substantial horizontal distance to the atmospheric volume probed by TURBO at $85 \mathrm{~km}$ : CUPRI looked in the vertical direction, whereas TURBO flew through the $85 \mathrm{~km}$ layer at a horizontal range of 13.3 and $51.9 \mathrm{~km}$ on upleg and downleg, respectively, nearly northward from CUPRI. The EISCAT VHF system is located in Troms $\varnothing\left(69.7^{\circ} \mathrm{N}\right.$, $19.0^{\circ} \mathrm{E}$ ), approximately $220 \mathrm{~km}$ from Esrange.

\section{Relative density fluctuations and PMSE}

In Figure 1 the radar echo strength measured by CUPRI is shown together with the relative percentage fluctuations of all 4 in situ measurements. More details about the CUPRI observations are presented in the companion paper of Cho et al. [1993]. We have averaged the CUPRI signal in the period \pm 20 minutes around the TURBO launch in order to account for the horizontal distance mentioned above: assuming $40 \mathrm{~m} / \mathrm{s}$ horizontal wind speed and arbitrary direction, the distance of $51.9 \mathrm{~km}$ corresponds to a minimum time lag of $\sim 20 \mathrm{~min}$. A strong PMSE is observed by CUPRI in the altitude range $84-91 \mathrm{~km}$. There is a tendency for a double layered structure which we use to distinguish between an 'upper' and a 'lower' PMSE layer throughout this paper.

The electron densities show strong fluctuations at the altitude of the lower PMSE layer and, to a lesser extent, also at the upper layer. The variability is small in the rest

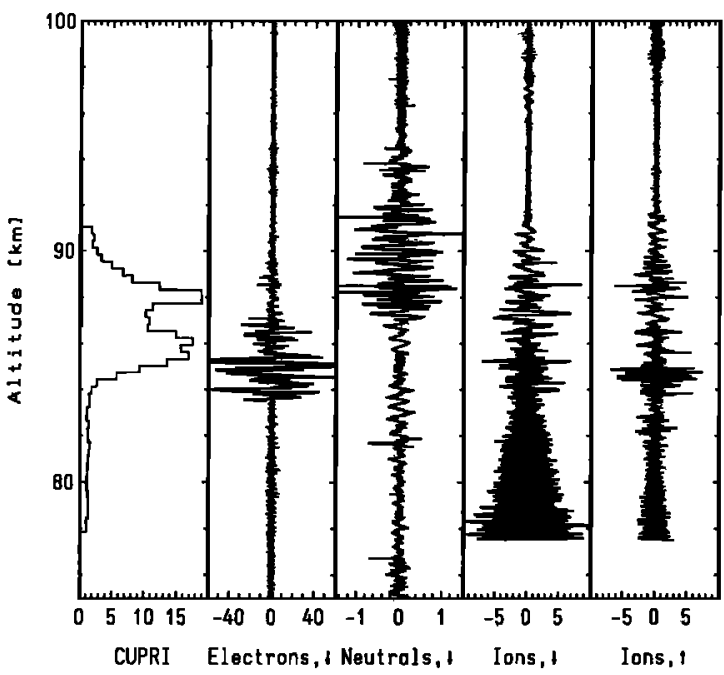

Fig. 1. S/N ratio from CUPRI and relative density fluctuations (in \%) simultaneously measured in situ in flight NBT5 of the NLC-91 campaign ( $\uparrow=$ upleg, $\downarrow=$ downleg). of the profile. The neutrals also show strong fluctuations but located at higher altitudes $(\sim 87-92 \mathrm{~km})$. Comparatively small variations are observed in the rest of the profile. Please note that the neutral residuals are extremely small at the altitude of the lower PMSE layer. The ions show fluctuations from $84-91 \mathrm{~km}$ both on upleg and on downleg (the increase in the lowest part is due to instrumental noise). We note that the two ion profiles show some similarities: the fluctuations are virtually absent above $91 \mathrm{~km}$ and both profiles show a tendency to a maximum around $85 \mathrm{~km}$. This suggests that the layer is horizontally stratified with a tilt smaller than 1-2 km / $40 \mathrm{~km}$.

In Figure 2 expanded views of the residuals in the lower and upper PMSE are shown (we have not plotted the ion density fluctuations on upleg, since the horizontal distance to the other profiles is too large). When comparing absolute magnitudes the scaleheight effect has to be taken into account: following Thrane and Grandal [1981] neutral and ion density fluctuations are related by the ' $F$-factor' $\left(\Delta n / n=F \cdot \Delta N_{i} / N_{i}\right)$ which is determined by neutral and plasma density scaleheights.

In Figure 2a strong fluctuations in the electron and ion densities are shown. An even closer look shows that significant fluctuations are present down to very small scales below 3 meters (see Figure 3). These type of 'spiky' fluctuations can explain the CUPRI radar echoes, provided the irregularities extend over at least a Fresnel zone ( $1 \mathrm{~km}$ ) [Hoppe et al., 1993]. We are also studying rocket
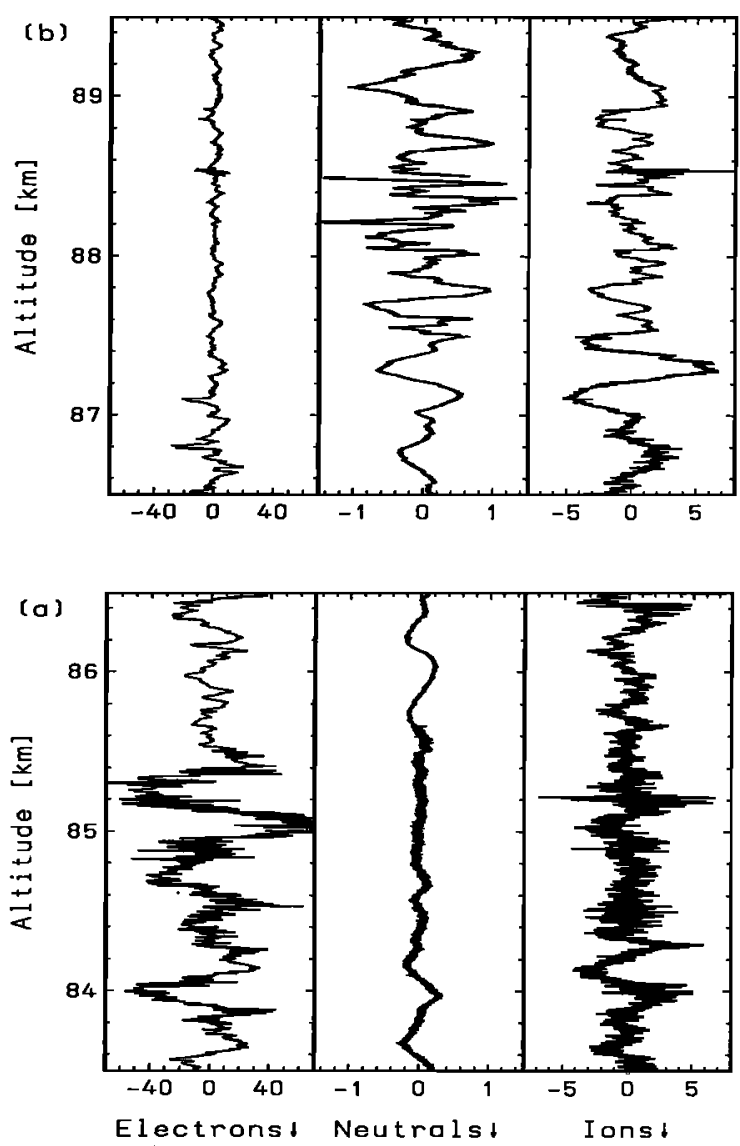

Fig. 2. Expanded view of the fluctuations shown in Figure 1 in the lower (a) and upper (b) PMSE layer. 
potential changes as a conceivable source of these fluctuations. A more detailed discussion on this aspect and on the difference in magnitude between electron and ion density fluctuations will be published in the near future. In remarkable contrast to the plasma fluctuations, the TOTAL instrument shows hardly any fluctuations at all.

Coming to the upper PMSE layer next (Figure 2b): all three profiles show a very different behavior compared to the lower layer: the electron fluctuations are much smaller and not so spiky, the ions exhibit strong medium scale variations but also without any spiky fluctuations and the neutrals show strong fluctuations. In fact, these fluctuations are among the strongest we have ever observed in 17 TURBO flights in the last 2 years. When comparing neutral and ion fluctuations, an anti-correlation is clearly visible both at large and medium scales. The F-factor measured in this altitude range was on the order of $-1 / 10$. We therefore observe exactly what is expected from turbulence theory: the ion fluctuations are anti-correlated to the neutrals and approximately an order of magnitude larger.

Although the difference in the neutral density fluctuations between the upper and lower layer is very striking we must be careful not to draw conclusions too early. The reason for this is that the conversion of turbulent motions to neutral density fluctuations depends on the background temperature profile. It can be shown that the 'sensitivity' of neutral density fluctuations as a passive tracer for turbulence is proportional to $\omega_{B}^{2}\left(\omega_{B}=\right.$ Brunt-Väisälä frequency). We have obtained $\omega_{B}^{2}$ from the combined temperature measurements of the falling spheres and of TOTAL. Indeed, lower values of $\omega_{B}^{2}$ were found in the lower PMSE layer compared to the upper layer $\left(\omega_{B}^{2} \sim 0.4 \cdot 10^{-3}\right.$ and $1.2 \cdot 10^{-3} / \mathrm{s}^{2}$, respectively). However, the difference is only a factor of $\sim 3$ which leaves enough sensitivity to detect turbulence in the lower PMSE layer.

\section{Spectra of density fluctuations and relationship to PMSE}

In order to gain further insight into the nature of the fluctuations in the two layers, we have studied the spectra of the residuals shown in Figure 2. We will again start our discussion with the lower layer. The spectrum (not shown here) of the neutral density fluctuations of Figure $2 a$ is very flat. We have compared this spectrum with theoretical spectra to be expected if there were turbulence present. We found that the measured power spectral densities (PSD) in the inertial subrange are almost two orders of magnitude smaller than any theoretical curve, even when we consider very weak turbulence $(\epsilon=1 \mathrm{~mW} / \mathrm{kg})$. We therefore conclude that the spectrum of neutral density fluctuations in the lower PMSE layer confirms that there is very little or no neutral turbulence present.

The electron and ion fluctuation spectra also show a nonturbulent spectrum. In Figure 3 we show an example derived from electron fluctuations in the lower layer (84 \pm $0.5 \mathrm{~km}$ ). This spectrum is not compatible with the passive scalar spectrum of Driscoll and Kennedy [1985] ('D\&K model') since no indication of an inertial subrange is observed. As can be seen from Figure 3, the power spectral densities at scales of $3 \mathrm{~m}$ are significantly above the noise level: the noise current of the electrometer zero-line is $\sim$ $10 \mathrm{pA}$ (RMS) which corresponds to a PSD of $5 \times 10^{-6}$ at

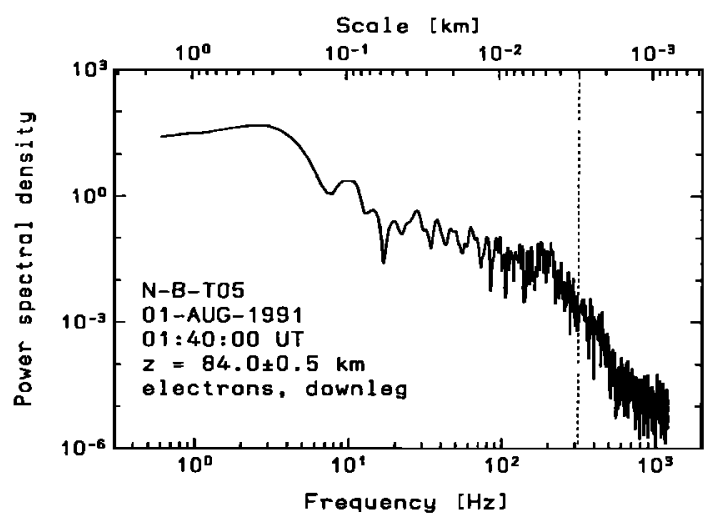

Fig. 3. Electron density fluctuation spectrum observed at $84 \pm 0.5 \mathrm{~km}$, which is within the lower PMSE layer. Frequencies have been converted to scales using $L=\lambda=v_{R} / \nu$ $\left(v_{R}=\right.$ rocket velocity $\left.=960 \mathrm{~m} / \mathrm{s}\right)$.

$84 \mathrm{~km}$, as is observed at very high frequencies in Figure 3.

Coming to the upper layer next: all indications suggest that there is turbulence present here. Apart from the anti-correlation between neutral and ion fluctuations mentioned above, the neutral density fuctuation spectrum nicely agrees with a theoretical model using $\epsilon \approx 83 \mathrm{~mW} / \mathrm{kg}$ (see Figure 2 in Lübken, 1992). When comparing this spectrum with that measured by the ion probe in the same height range (taking into account the $F$-factor) we find good agreement at large and medium scales. However, at larger wavenumbers the ion spectrum shows larger PSD compared to the neutral spectrum. This is expected if the diffusivity of the ions is significantly reduced and the Schmidt number is larger than 1 . We have estimated $S c$ by fitting the $D \& K$ model to the measured ion spectrum and found $S c \approx 3$. We note, however, that the measured spectrum is not very well represented by a D\&K model (the latter falls off too rapidly).

We now compare the observed electron fluctuations with the CUPRI radar echo power. The electron fluctuations are much smaller in magnitude in the upper layer compared to the lower layer, but the CUPRI echo power is approximately the same. This can be understood if we estimate

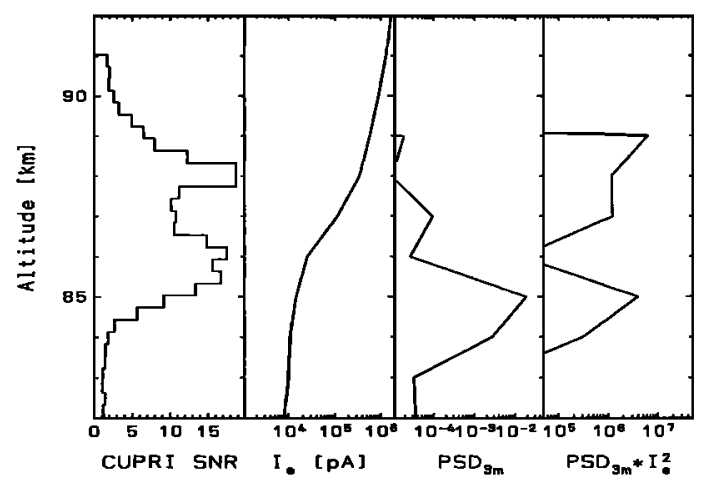

Fig. 4. From left to right: CUPRI $S / N$ ratio ; electron current ; power spectral density of the electron fluctuations averaged over scales from $3 / 1.25$ to $3^{*} 1.25$ meters ; product $P S D_{3 m} \cdot I_{2}^{2}$. According to theory the latter quantity is proportional to the radar backscattered power. 
the relative echo power strength: the $S / N$ ratio observed by the radar is proportional to the power spectral density at $3 \mathrm{~m}$ scale $\left(P S D_{3 m}\right)$ times $N_{e}^{2}\left(N_{e}=\right.$ electron number density) [Ulwick et al., 1988] (this assumes, however, that the radar backscatter volume is filled with scatterers). As can be seen from Figure 4, $P S D_{3 m}$ is much smaller in the upper layer compared to the lower layer, however, the total electron current increases by a factor of 30 between 84 and $88 \mathrm{~km}$. Over a short altitude range we can assume that the sensitivity of the electron probe is nearly constant and use the electron current $I_{e}$ as a measure of the electron density $N_{e}$. The height profile of the product $P S D_{3 m} \cdot I_{e}^{2}$ in Figure 4 shows that indeed the radar echoes should be of similar magnitude in both layers, as is observed by CUPRI.

\section{Discussion and Conclusions}

Further evidence for a fundamentally different backscatter process comes from the aspect sensitivity of the CUPRI echo [Cho et al., 1993]. In addition, the spectral width of the EISCAT VHF radar echo is also different at both layers: the spectrum received from $86 \mathrm{~km}$ has a width of 1 $\mathrm{Hz}$ (FWHM), the one from $89 \mathrm{~km}$ is $11 \mathrm{~Hz}$ wide and a factor of 2-3 less intense. Assuming that this width is entirely given by turbulence the turbulent velocity becomes $w^{\prime}=1.8 \mathrm{~m} / \mathrm{s}$. This agrees very well with the $\boldsymbol{w}^{\prime}=2.2 \mathrm{~m} / \mathrm{s}$ that is derived from $\epsilon=83 \mathrm{~mW} / \mathrm{kg}$ given above.

In conclusion, we find that our in situ observations of neutral and plasma fluctuations during a PMSE event reveal the coexistence of different backscattering mechanisms in different parts of the PMSE layer: we have shown that the echoes from the lower part of the PMSE layer are not caused by neutral air turbulence. Some other nonturbulent process must have created the fine structures in the electron and ion gas (and must support it against destruction by diffusion). Simultaneously, in the upper part of the PMSE layer we find conclusive indications for neutral turbulence. Regardless of their creation mechanism, our estimate of the relative backscattered power shows that the electron fluctuations at $3 \mathrm{~m}$-scale lead to approximately the same echo strength in both parts of the PMSE layer, as is observed.

Acknowledgements. We gratefully acknowledge the help from Prof. U. von Zahn and F. Föhner. The thank the personnel from ESRANGE, MORABA, NASA and NDRE for their excellent work. This study was supported by the BMFT, Bonn under grant 010E88027. The CUPRI system and the Cornell team were supported by NASA and by NSF. The EISCAT Scientific Association is supported by CNRS (France), SA (Finland), MPG (Germany), NAVF (Norway), NFR (Sweden) and SERC (United Kingdom).

\section{References}

Blix, T. A., E. V. Thrane, and O. Andreassen, In situ measurements of fine scale structure and turbulence in the mesosphere and lower thermosphere by means of electrostatic positive ion probes, J. Geophys. Res., 95, 5533-5548, 1990.

Cho, J., W. Swartz, and C. Miller, The characteristics of PMSE during Salvo B of NLC-91: evidence of partial reflection and turbulent scatter, (this issue), 1993.

Driscoll, R. J., and L. A. Kennedy, A model for the spectrum of passive scalars in an isotropic turbulence field, Phys. Fluids, 28, 72-80, 1985.

Ecklund, W. L., and B. B. Balsley, Long-term observations of the arctic mesosphere with the MST Radar at Poker Flat, Alaska, J. Geophys. Res., 86, 7775-7780, 1981.

Hillert, W., F.-J. Lübken, and G. Lehmacher, Neutral air turbulence during DYANA as measured by the TOTAL instrument, J. Atmos. Terr. Phys., submitted, 1992.

Hoppe, U.-P., C. Hall, and J. Röttger, First observations of summer polar mesospheric backscatter with a 224 MHz radar, Geophys. Res. Lett., 15, 28-31, 1988.

Hoppe, U.-P., T. A. Blix, E. V. Thrane, and F.-J. Lübken, Studies of polar mesosphere summer echoes by VHF radar and rocket probes, J. Atmos. Terr. Phys., (submitted), 1993.

Kelley, M. C., D. T. Farley, and J. Röttger, The effect of cluster ions on anomalous VHF backscatter from the summer polar mesosphere, Geophys. Res. Lett., 14, 1031-1034, 1987.

Lübken, F.-J., On the extraction of turbulent parameters from atmospheric density fluctuations, $J$. Geophys. Res., 97, 20,385-20,395, 1992.

Providakes, J. F., W. E. Swartz, D. T. Farley, and B. G. Fejer, First VHF auroral radar interferometer observations, Geophys. Res. Lett., 10, 401-404, 1983.

Röttger, J., and C. LaHoz, Characteristics of polar mesosphere summer echoes (PMSE) observed with the EISCAT $224 \mathrm{MHz}$ radar and possible explanations of their origin, J. Atmos. Terr. Phys., 52, 893-906, 1990.

Röttger, J., C. LaHoz, M. C. Kelley, U.-P. Hoppe, and C. Hall, The structure and dynamics of polar mesosphere summer echoes observed with the EISCAT 224 MHz radar, Geophys. Res. Lett., 15, 1353-1356, 1988.

Thrane, E. V., and B. Grandal, Observations of fine scale structure in the mesosphere and lower thermosphere, J. Atmos. Terr. Phys., 43, 179-189, 1981.

Ulwick, J. C., K. D. Baker, M. C. Kelley, B. B. Balsley, and W. L. Ecklund, Comparison of simultaneous mst radar and electron density probe measurements during state, J. Geophys. Res., 93, 6989-7000, 1988.

F.-J. Lübken and G. Lehmacher, Department of Physics, Bonn University, Nussallee 12, 5300 Bonn, Germany.

T. Blix, U.-P. Hoppe and E. Thrane, Norwegian Defence Research Establ., P.O. Box 25, 2007 Kjeller, Norway

J. Cho and W. Swartz, Cornell University, School of Electrical Engineering, Ithaca, New York, 14853
(Received October 23, 1992; revised February 5, 1993; accepted February 23, 1993) 